\title{
Development of AHTS-vessel Marine Engine Room Virtual Training Platform and Research on Key Technology
}

\author{
Haosheng Shen ${ }^{1}$, Jundong Zhang ${ }^{1 *}$, Hong Zeng ${ }^{1}$ and Yuanyuan Tang ${ }^{2}$ \\ ${ }^{1}$ Marine Engineering College, Dalian Maritime University, China \\ shen7231591@126.com
}

\begin{abstract}
"HYSY681" is selected as the parent ship to develop the AHTS (Anchor Handling Tug Supply) vessel MERVTP (Marine Engine Room Virtual Training Platform). Hybrid simulation design mode is applied to the training platform and the advantage of each applied simulation method is fully made. In order to give consideration to both the instantaneity and accuracy required by 2D-MERSS (2D Marine Engine Room Simulation Software), simulation algorithm and strategy are analyzed and chosen reasonably. To improve the efficiency of development and expansibility, the simulation data is planed from the whole prospect, WPF (Windows Presentation Foundation) technique is applied to the development of 2D-HMI (2D Human-machine Interface) and the refresh mechanism of $2 \mathrm{D}-\mathrm{HMI}$ is optimized to improve the simulation efficiency. In order to promote the overall training effect, visual simulation technique is applied to this training platform and 3D-MERSS (3D Marine Engine Room Simulation Software) is developed. Optimization techniques including LOD (Level of Detail), structural nodes optimization, Prefab and rendering method based on Shader are adopted to promote the user experience. To further enhance the immersion, 3D-MERSS is also applied to the 3channel stereo projection environment, meanwhile, the relevant techniques including scene segmentation, edge blending and geometric correction are analyzed and researched.
\end{abstract}

Keywords: AHTS vessel, Marine Engine Room, HMI, Visual Simulation, 3-channel Stereo Projection

\section{Introduction}

With the rapid development of global economy, the quantity demanded of energy source continues to increase and the energy problem is becoming more and more serious every day. There is affluent petroleum resource in the coastlines and shallow continental shelves and the offshore petroleum will become the main part of the future energy source for all mankind doubtlessly. The AHTS vessel plays an important role during the exploitation of offshore petroleum. Compared with the conventional merchant vessels such as VLCC, container carrier and bulk cargo carrier, the AHTS vessel needs to undertake various kinds of offshore operations, so it puts forward higher requirements on the marine engineers.

Excellent work ability depends on systematic training to a large extent and better training effect for the marine engineers can be achieved through MERS (Marine Engine Room Simulator), meanwhile, the "STCW 78/10" convention amendment, coming into effect in March, 2012, has also ruled clearly that the marine engineers must got trained through MERS. Therefore, it has a great significance to develop a marine engine room virtual training platform aiming at the AHTS vessel. At present, the famous MERS manufacturers at home and abroad includes KONGSBERG in Norway, TRANSAS in England, UNITEST in Poland, K-LINE in Japan, Wuhan University of Technology, Shanghai Maritime University and Dalian Maritime University in China [1], the MERS 
developed by them are applied widely in many maritime colleges and training institutions in the world and satisfactory training results are achieved. However, the current MERS mainly focuses on conventional merchant vessels such as $V L C C$, container carrier, bulk cargo carrier and the MERS aiming at AHTS vessel is rare.

At the moment, most of the mainstream MERS are developed based on 2D and semiphysical simulation. Let's take the "Neptune" MERS developed by KONGSBERG as an example [2], it takes the $2 D$-MERSS as the core unit to calculate the math model in real time and simulation data is transmitted between $2 D$-MERSS and physical consoles by establishing communication protocol, meanwhile, a certain number of trainee stations are equipped. Design mode like this mainly focuses on the studying of marine engineering system principle and operation method, however, the training environment is very different from the real marine engine room both in vision and auditory feeling and it limits the training effect through MERS.

In recent years, with the maturity of visual simulation technique, it provides a new way for the education and training in every field, meanwhile, it also provides a new idea for the development of modern MERS. Therefore, many MERS manufacturers have tried to introduce the visual simulation technique into the traditional MERS, for example, the LER3D, MED3D, MER3D and PSV3D developed by UNITEST [3], K-SIM by KONGSBERG [4] and WMS2009 by Wuhan University of Technology [5]. However, after experiencing the existing $3 D-M E R S S$, it is found that the virtual scene is not real enough and the human-machine interaction content is not abundant enough, meanwhile, the manifestation mode is mainly based on desktop $V R$ (Virtual Reality), which limits the advantage of visual simulation technique.

Based the above-mentioned research status, to meet the special requirements on the marine engineers for AHTS-vessel and promote the training effect of the traditional MERS, "HYSY681" is selected as the parent ship to develop the AHTS-Vessel MERVTP in this paper. The developing process is introduced and the related key techniques are analyzed and researched in detail.

\section{Platform Architecture}

\subsection{Selection of the Parent Ship}

To guarantee the advancement and representativeness of the AHTS-Vessel MERVTP, the Chinese first high-horsepower AHTS-Vessel "HYSY681" is selected as the parent ship as the Figure.1 shows. "HYSY681" fills in the technology gap in the field of AHTS-vessel for China and thus it is very representative as the parent ship [6].

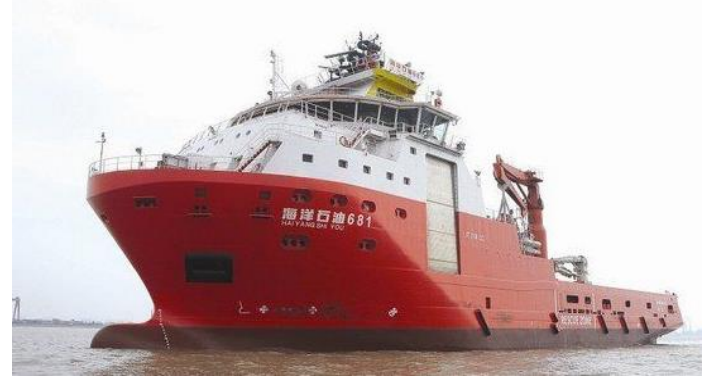

Figure 1. Ahts-Vessel "Hysy 681" 


\subsection{Design Principle}

According to the special requirements on the marine engineers in the AHTS-vessel and to promote the training effect of traditional MERS, the AHTS-Vessel MERVTP should follow the following design principles [7-8]:

(1) As the core unit of the whole AHTS-Vessel MERVTP, the math model of the marine engineering system should be precise enough and be able to reflect the running state of it veritably, particularly, extra math models should be established for the special marine engineering systems such as the dry bulk system, base oil system, cargo fresh water system and ballast/drilling water system for the AHTS-vessel.

(2) Plan the simulation data structure from the whole prospect, choose proper simulation algorithm and strategy and refresh mechanism so as to give consideration to both the instantaneity and accuracy required by $2 D$-MERSS.

(3) The 3D-MERSS should be up to standard in the aspects of 3D models, virtual environment, simulation data, graphics quality, sound effect, etc., in the meantime, the loading speed, interaction delay and fluency of scene graphics should also satisfy the users.

(4) Apply the 3D-MERSS to 3-channel stereo projection environment to take full advantage of the visual simulation technique.

\subsection{Platform Structure}

The AHTS-Vessel MERVTP is composed of master simulation station (coach station), physical simulation consoles, large-scale simulation mimic panel, child simulation stations, 3-channel stereo projection environment and a certain number of trainee stations. The structure of the training platform is as shown in Figure.2 [9].

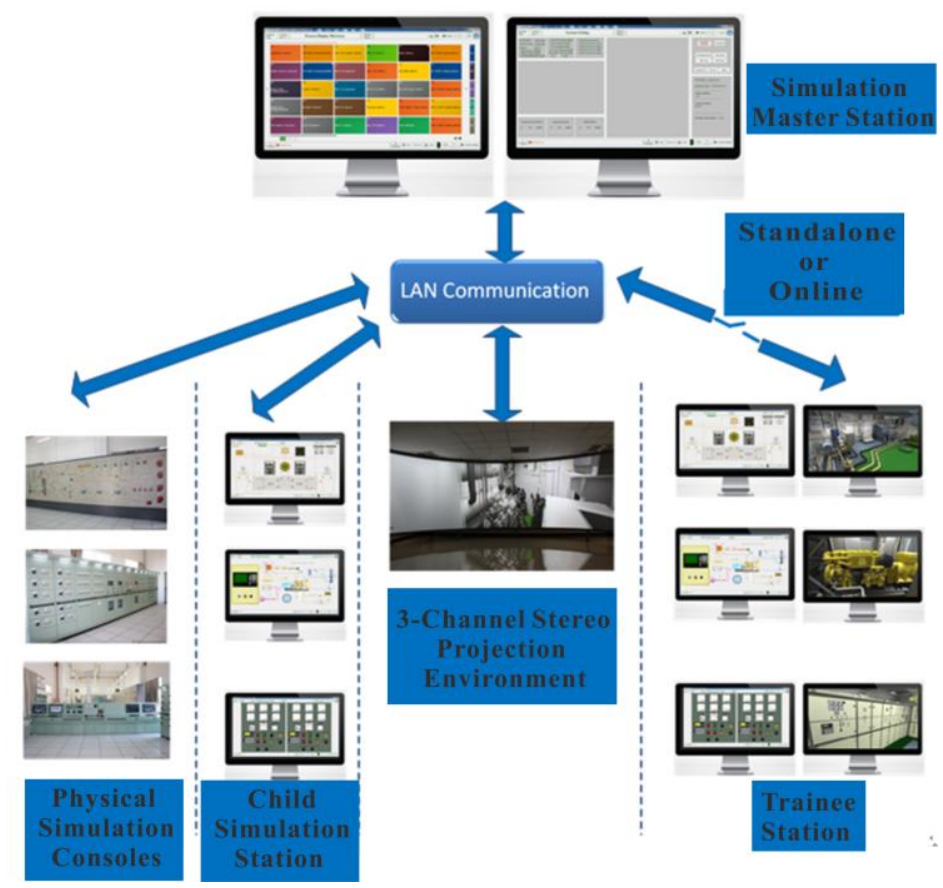

Figure 2. Structure of the AHTS-Vessel Marine Engine Room Virtual Training Platform

As the core unit of the training platform, the $2 D$-MERSS is running in the master simulation station and in charge of the real-time calculation of the math model of marine engineering system, meanwhile, the real-time simulation data calculated by $2 D$-MERSS is transmitted to the other parts to update the running state by establishing LAN (Local Area 
Network) communication system, in addition, coach station is also integrated in the master simulation station and it can achieve a certain number of coach functions such as state monitoring, fault setting, evaluation setting and state saving/loading; Physical simulation consoles (e.g. Engine Control Console, Bridge Control Console, Switchboard and Local Control Box) are designed and manufactured completely in accordance with the real ones so as to reproduce the real working environment; Large-scale simulation mimic panel entirely displays the pipe principle of the marine engineering system, the valves can be opened or closed and the main electromechanical equipment can be started or stopped by operating the buttons, meanwhile, the state parameters such as temperature, pressure and liquid level can be displayed dynamically through the dial meter and bargraph meter; Through analyzing the function and feature of each marine engineering system in 2D-MERSS, the whole system can be artificially divided into a number of subsystems such as cargo system, fuel system and fresh water system, which are respectively running in the child simulation station; The 3-channel stereo projection platform provides a training environment with high immersion and it can promote the trainees' perceptual cognizance on the real marine engine room environment; The configuration of trainee stations is relatively flexible, which permits the trainees to train solely by running the standalone version $2 D$-MERSS and $3 D$-MERSS jointly or train in a training team by connecting with the master simulation station.

The math model only runs in the master simulation station, the other parts only receive the real time simulation data from it to update the running state and send operation information to it to artificially interpose the calculation process of the math model, so the running state of the whole training platform can keep synchronous, which lays the foundation for the multi-user collaborative training mode.

\section{Development of the 2-D Marine Engine Room Simulation Software}

The 2D-MERSS is the core unit of the whole AHTS-Vessel MERVTP and the running state, changing trend of the running parameters all depend on the real-time calculation of the math model integrated in the $2 D$-MERSS, meanwhile, the HMI of the $2 D$-MERSS is in charge of several functions such as simulation operation, simulation data display, parameter setting, network connection, so the development of $2 D-M E R S S$ can be divided into the development of the math model simulation program and the HMI simulation program.

\subsection{Selection of the Parent Ship}

The math model should be able to reflect the arithmetical relationship of the marine engineering system truly and accurately, and possess good data structure, optimized simulation algorithm and reasonable simulation strategy so as to guarantee the requirements on veracity and instantaneity. As the marine engineering system is overly miscellaneous and enormous, this paper only introduces the planning of simulation data and the selection of simulation algorithm and strategy in detail rather than the mathematical modeling process.

\subsubsection{Planning of Simulation Data}

The planning of simulation data means the definition and organization of the simulation data such as pressure, temperature and flow.

According to the usage of the simulation data, they can be divided into public data and private data. The private data is used to the internal calculation of subsystem simulation program and the definition and organization of it is relatively flexible; The public data is used to update the running state of the whole training platform and to be invoked by other subsystem simulation program, so they must be planned from the whole prospect so as to 
facilitate identification, management, invoking and transmitting. The planning detail is as following:

The variable name of the public data is composed of four fields: Data Type + Identification of Marine Engineering Subsystem + Data Identification + Description.

The first field describes the data type of the public data, $F$ (Float) is used for the continuously changed simulation data such as pressure, voltage and flow, $B$ (Boolean) for the simulation data that only has two states (True or False) and $I$ (Integer) for the simulation data that has multiple discrete states.

The second field describes which marine engineering subsystem the public data belongs to by using identification whose range is from $0-99$, for example, 10 represents the fuel oil supply system.

The third field describes the identification of the public data whose range is from 000999 , and in this way the second and third field jointly forms the unique data identification in the simulation data class.

The forth field describes the actual physical meaning of the public data, which can be defined by the developer himself.

For example, the public data "F10189_DOTKLevel" represents the level of diesel oil tank

To take the public data "F10189_DOTKLevel" as example, it represents the level of the diesel oil tank, the data type is Float and the unique data identification is 10189.

\subsubsection{Simulation Algorithm and Simulation Strategy}

Compared with some precise simulation systems, the AHTS-Vessel MERVTP has a higher requirement on instantaneity and demands the simulation results to satisfy precision range to some extent, so both instantaneity and accuracy are given consideration when selecting and designing the simulation algorithm and strategy.

The calculation of the math model of marine engineering system mainly involves the solution of the differential equation and algebraic equation, and the solution of logical model. So far, the solution method of differential equation as the Eq.1 shows includes 1-4 order Adams-Bashforth $(A B)$ algorithm and real-time Runge-Kutta (RTRK) algorithm [10].

$$
y^{\prime}(t)=f(y(t), u(t)), y\left(t_{0}\right)=y_{0}, y \in R^{n_{S}+n_{N}}, t \in\left[t_{0}, T\right]
$$

The real part of the numerical stability region for the $A B$ algorithm is as follows:

The Euler algorithm is $|h \lambda|<2, A B 2$ algorithm is $|h \lambda|<1, A B 3$ algorithm is $|h \lambda|<0.55$, $A B 4$ algorithm is $|h \lambda|<0.3$

The real part of the numerical stability region for the $R T R K$ algorithm is as follows:

The RTRK1 algorithm is $|h \lambda|<2$, RTRK2 algorithm is $|h \lambda|<2, R T R K 3$ algorithm is $|h \lambda|<2.5$, RTRK4 algorithm is $|h \lambda|<2.785$.

Apparently, the RTRK algorithm has a larger numerical stability region, which is suitable for the solution of weak rigidity system, but it needs more calculation times for the function in the right side and sampling times every integration step, especially the high-order $R T R K$ algorithm. So in order to satisfy the requirement on instantaneity, this paper applies $R T R K 3$ algorithm to the marine engineering subsystem that has a lower requirement on the data precision and stability and $R T R K 4$ algorithm is applied to the subsystem that has a higher requirement on the data precision and stability. But for the strong rigidity local system, Euler algorithm should be applied. In addition, different integration step is applied based on the subsystem's time constant.

\subsection{Human-Machine Interface}


As the major carrier of simulation operation and data presentation, $H M I$ is the external manifestation of the quality of $2 D-M E R S S$ and it is required to be elegant and pretty on the basis of not losing expertise, meanwhile, the human-machine interaction function should be easy to use and it can also motivate the enthusiasm of operating for the users. In order to guarantee the expertise and consistency of the HMI, the design style is planed from a global perspective as following:

(1) Uniformity: the color of the pipes, valves and other elements in the interface must conform to the RGB value indicating the different fluid medium regulated by the CCS (China Classification Society) "Classification Rules of Steel Sea-going Vessel", meanwhile, the thickness of the pipe and the interaction elements such as valves, button and switch in the $H M I$ must be uniform.

(2) Consistency with the Real Ship: The pipe system, the control consoles and panels in the HMI must be designed and drew completely based on the construction drawings so as to keep consistent with the real ship.

(3) Users' Experience: The HMI must be designed as user-centered based on the principle of ergonomics and usability engineering and synthesize vision, layout, interaction behavior and other design methods so as to conform with users' operation habit.

\subsubsection{Development Technique}

Vector graph drawing software, CorelDraw, is applied in the drawing of $H M I$ in this paper. WPF (Windows Presentation Foundation) instead of GDI+ (Graphics Device Interface+) is adopted to develop the interaction elements such as valves, meters and buttons in the $H M I$ in this paper, this is because the developer must manually write a lot of code to implement coordinate transformation, color filling, outline drawing and event trigger by using $G D I+$, however, $W P F$ can achieve the same effect only with a small number of code and most of the code is generated automatically [11], so the developing efficiency is promoted drastically and the expansibility of the $H M I$ is enhanced.

As the $2 D$-MERSS should undertake the real-time calculation of the math model, which will consume a certain of computer resource, so in order to meet the requirement on instantaneity, the running and refreshing mechanism is optimized when developing the $H M I$ as follows:

(1) Separate the simulation program of math model and $H M I$ and adopt different refreshing clock.

(2) Event trigger mechanism is used to deal with the human-machine interaction instead of the traditional looping query mechanism.

(3) Apply the multi-document window structure. When a certain sub-window is running and activated, destroy all the inactivated sub-windows and only refresh the interaction elements in the current activated sub-window.

\subsubsection{Examples of the HMI}

The Figure. 3 is the $H M I$ of the main propulsion power system. Compared with the regular merchant vessel, the AHTS-vessel has a higher requirement on the maneuver capability, so "HYSY 681" is equipped with two main propulsion diesel engines, three tunnel thrusters and two azimuth thrusters. The users can enter into the corresponding $H M I$ by clicking the button in the left side, and then conduct simulation operations such as the starting or stopping of the thruster, transformation of control position and observe the main parameters such as speed, pitch and load. 


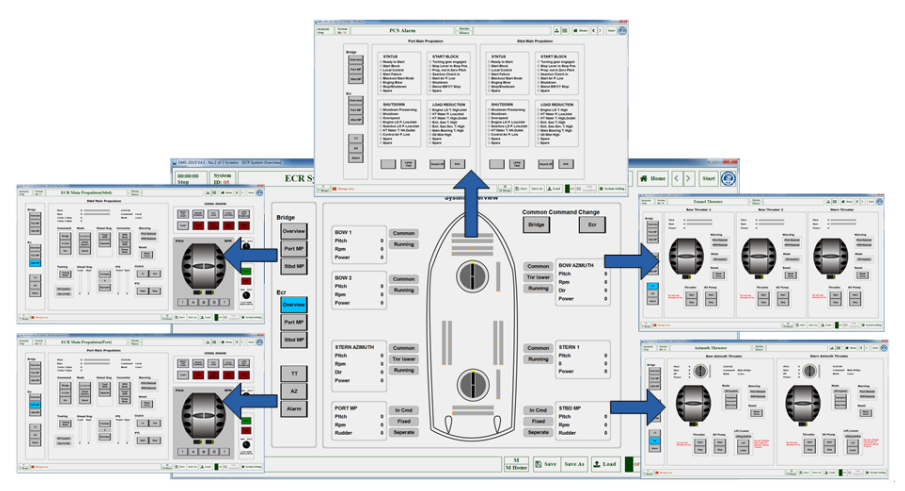

Figure 3. HMI of the Main Propulsion Power System

The Figure. 4 is the $H M I$ of the dry bulk system, which can simulate the integrated handling process of the dry bulk and display main state parameters such as the level of dry bulk tank, pressure of compressed air and instantaneous flow of the dry bulk inlet and outlet pipe.

The Figure. 5 is the $H M I$ of the fuel oil cargo system, which can simulate the integrated handling and transforming process of the fuel oil and display main state parameters such as the level of fuel oil tank, the suction and discharge pressure of fuel oil pump and the instantaneous mass flow of main pipe.

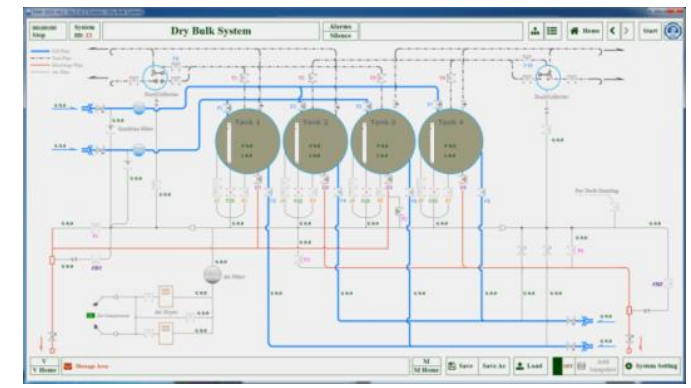

Figure 4. HMI of the Dry Bulk System

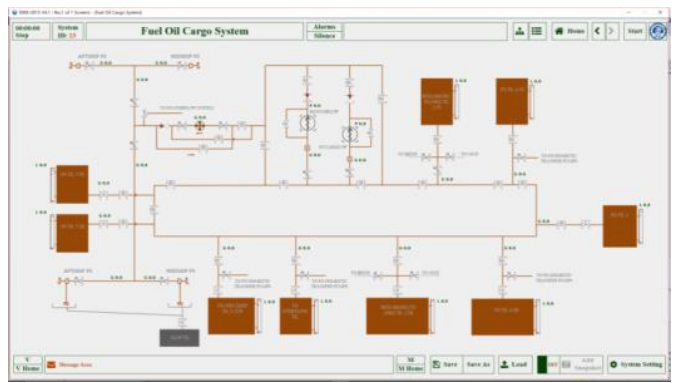

Figure 5. HMI of the Fuel Oil Cargo System

The other typical marine engineering systems of the AHTS-vessel includes base oil system, brine system, mud system, cargo tank washing system, etc. and the development of the above-mentioned systems' simulation program can help the trainees to master the working principle and operation procedure of the marine engineering system of the AHTS-vessel roundly. In view of the limitation of paper length, the other HMI of marine engineering system are not introduced in detail.

The Figure.6 is the coach management interface, which is mainly used to control and manage the training process. The specific functions of the coach management interface mainly include network parameter setting, record and print, evaluation setting, etc. In 
addition, the coach can set faults during the training and evaluation process by motivating a certain fault in the fault list and then freeze the fault when the trainee finds out the fault source. The Figure.7 is the fault setting list of the starting compressor system and faults such as the filth blockage of the cooler, valve leakage, motor failure and piston ring leakage can be set.

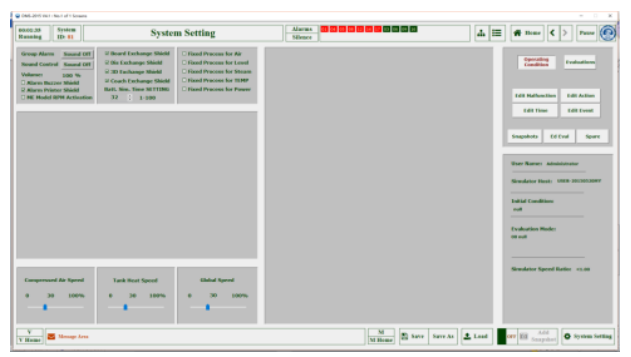

Figure 6. HMI of Coach Management

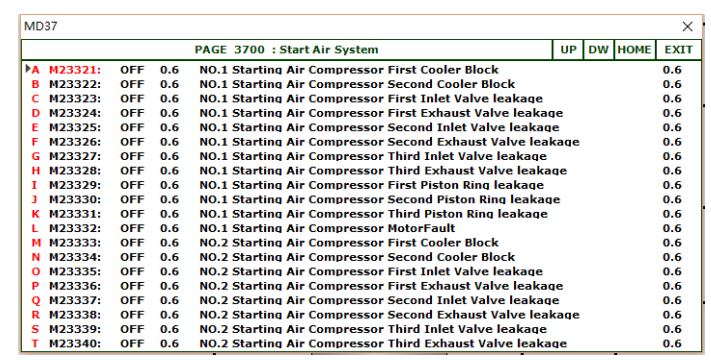

Figure 7. HMI Fault Setting

\subsection{Network Communication Program}

In order to implement the two-way interaction between the 2D-MERSS and the physical simulation consoles, a data collection module as the Figure. 8 shows is developed, which takes the embedded single-chip computer as the core processor and is used for the collection, conversion and transmission of the simulation data and operation information and UDP communication protocol is adopted. Each of the data collection modules has a unique IP address and it can process 16 analog quantities including 8 inputs and 8 outputs, and 64 switching quantities including 32 inputs and 32 outputs. The format of the $U D P$ communication protocol is as follows:

(1) The communication protocol format from $2 D$-MERSS to the data collection module

The beginning character $(0 x F E)+$ total data length + channel number + channel value $+\ldots+$ checkout + ending character $(0 \mathrm{xFF})$. As the size limitation of a single data package is 60 bytes, so the communication data can be sent after splitting if exceeded. The channel numbers are from $0 \times 10$ to $0 \times 2 \mathrm{~F}$ stands for the associated switching quantities and the channel numbers are from 0x00 to 0x07 for the associated analog quantities.

(2) The communication protocol format from the data collection module to $2 D-M E R S S$

The data collection module sends 32 switching quantities to the $2 D$-MERSS and the communication format is: the beginning character $(0 \mathrm{xFE})+$ channel number + channel value + checkout + ending character $(0 \mathrm{xFF})$, the channel numbers are from $0 \mathrm{x} 30$ to $0 \mathrm{x} 4 \mathrm{~F}$ for the associated switching quantities. 

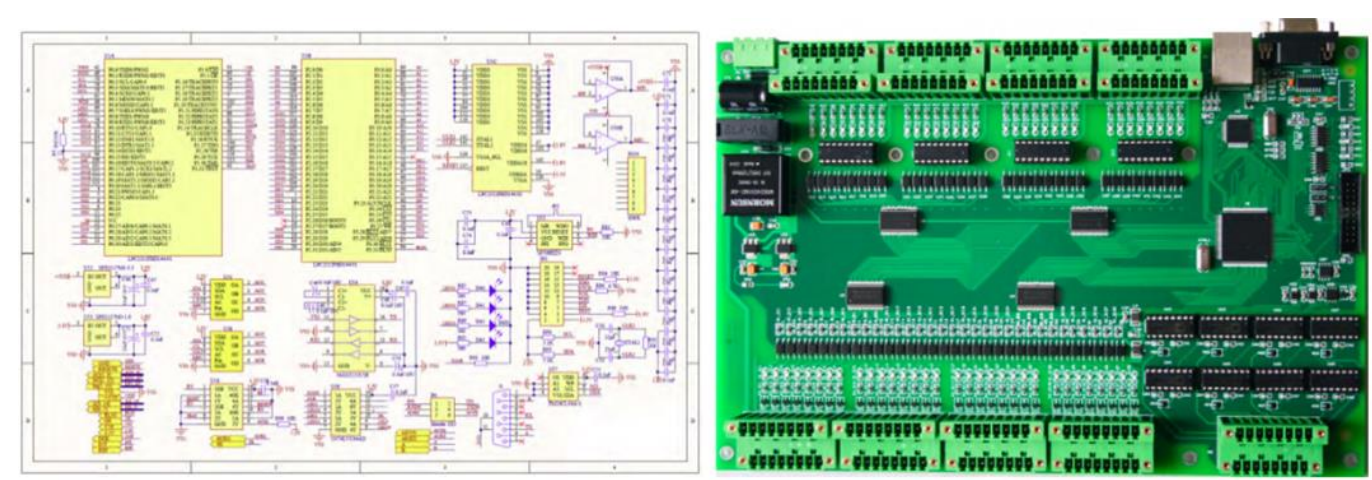

Figure 8. PCB Design Drawing and Physical Map of the Data Collection Module

The communication data between the data collection module and 2D-MERSS are analog quantity and switching quantity. The switching quantity will be only sent when it changes and the analog quantities will be only sent when its changing range meets the minimum precision of engineering value, in this way it can guarantee the efficiency and speediness of the network communication process. In addition, it is inevitable that packet dropout happens during the communication process, which will cause the loss of data, however, the periodical refreshing mechanism of the $2 D$-MERSS will update the simulation data automatically and guarantee the accuracy of communication process.

The high level programing language $\mathrm{C} \#$ based on the development platform Visual Studio 2013 is used to develop the $2 D$-MERSS and the running procedure is as the Figure. 9 shows. When $2 D$-MERSS starts to run, it will ask the user to load the initial running state and the user can choose to load a saved state or the default dead-ship state. In addition, $2 D$-MERSS will inquire the user if they want to train jointly with the physical simulation consoles or the $3 D$-MERSS so as to judge whether or not to run the network communication program. As the Figure.10 shows is the running procedure of the simulation program of marine engineering subsystem. According to the running feature and the different requirements on the instantaneity and veracity, simulation program of marine engineering subsystem adopts different simulation algorithm, strategy and refreshing period. 


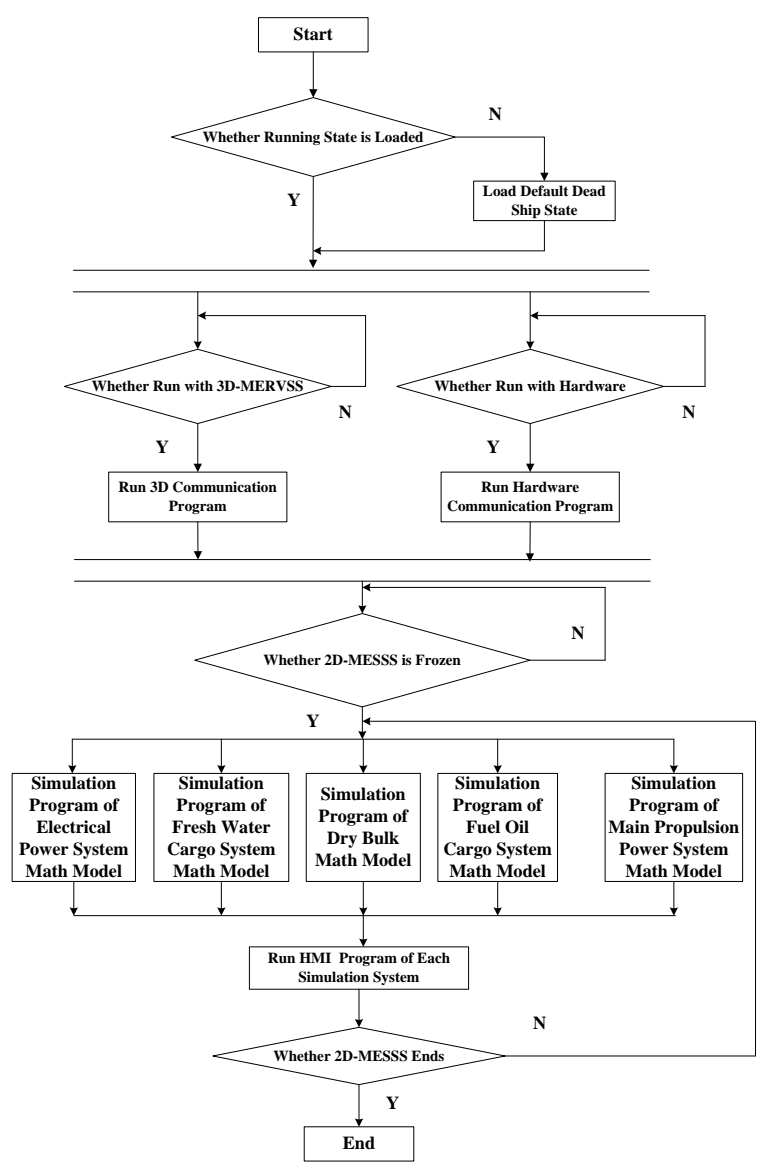

Figure 9. Running Procedure of 2D-MESSS

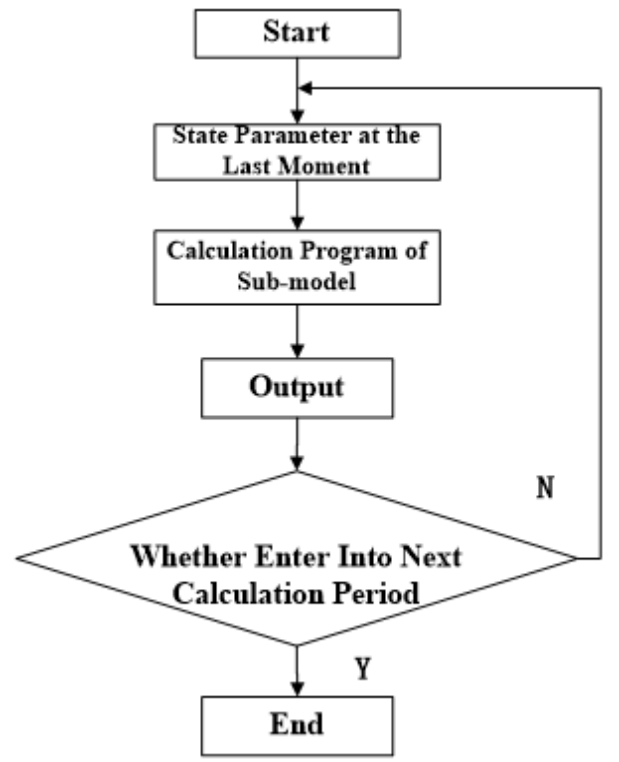

Figure 10. Running Procedure of Simulation Program of Marine Engineering Subsystem 


\section{Development of the 3-D Marine Engine Room Simulation Software}

In order to overcome the disadvantage that the training environment of the traditional marine engine room simulator is largely different from the working environment in the real ship, this paper utilizes the $V R$ technology to establish the 3D-MERSS: 3D-Max is used to establish the geometric model of entire marine engine room, electromechanical equipment and the pipe system strictly based on the image data of the parent ship, instruction book of the equipment and the principle drawings of the marine engineering system; 3D engine Unity-3D is used to develop the $3 D$-MERSS. The $3 D$-MERSS is also applied in the 3-channel stereo projection environment, which greatly enhances the immersion.

\subsection{Optimization Techniques for the 3D Marine Engine Room Virtual Software}

One important goal of $V R$ is to provide the user an objective and real virtual scene, which is also the foundation for developing high quality $3 D$-MERSS. However, pursing the realistic of the virtual scene excessively will increase the model size of the virtual scene drastically and influence the loading speed, fluency of the graphics and interaction delay, so in order to give consideration to both the virtual scene and the performance property, optimization techniques including LOD (Level of Detail), structural nodes optimization and Prefab are adopted.

\subsubsection{LOD (Level of Detail)}

The basic idea of $L O D$ [12]: If a virtual scene is descripted by the geometric model with multi-level details, that is to say, the object in the virtual scene has multiple corresponding geometric models and the difference among them is the description degree of the object's detail. When the virtual scene is rendering in real time, the object's model with simple detail can be used to promote the rendering speed. The selection of object's model with multi-level details depends on the object's degree of importance, which depends on the object's occupation area in the graphics space and other various factors.

As the marine engine room belongs to large-scale scene, the scene content that can be seen by the users is limited in one time and the object elements in the field of view have different distances to the users. So $L O D$ can be used to adjust the level of the object element's detail in real-time rendering: The model with low level detail can be used to promote the rendering speed for the object element far from the users; The model with high level detail can be used to enhance the realistic and promote the users' experience for the object element closed to the users.

At present, the $L O D$ technique is divided into static $L O D$ and dynamic $L O D$. This paper chooses static $L O D$ in view of the requirement on instantaneity by $3 D-M E R S S$, which achieves the smooth transition of $L O D$ models by establishing several geometric models with different level details in advance. The static $L O D$ can save lots of computer resource used to generate the real-time $L O D$ models and thus the simulation efficiency of the $3 D-M E R S S$ can be promoted.

This paper adopts the SOFM (Self-Organizing Feature Map) region segmentation algorithm to generate the static LOD models with multi-level details [13]. This algorithm segments the geometric model in advance based on giving considerations to the detail feature of it, which will make the simplification process controllable. The specific procedure of this algorithm is as follows:

Step 1: The model is segmented into several regions based on the SOFM region segmentation algorithm and the global merging scale $D$ is determined according to actual demand.

Step 2: The merging weight of the region $i$ is determined according to the proportion of the vertex number $N_{i}$ of the region $i$ in the vertex number $M$ of the whole model and the merging scale in region $i$ is determined as the Eq.2: 


$$
E_{i}=D N_{i} / M
$$

Step 3: Calculate the linear average distance $d_{i}$ between the each vertex in the region $i$ and the surrounding adjacent vertex of it, and regroup the region $i$ by deleting the vertex with the maximal $d_{i}$.

Step 4: Go into the step 5 if the merging scale of the region $i$ reaches the corresponding merging scale $E_{i}$, or return back to Step 3 .

Step 5: Exit the algorithm if all the regions complete merging, or return back to Step 2.

This paper selects the $L O D$ model with different level details based on the distance between the object model and the viewpoint and different number of $L O D$ model is set for every object model based on its importance, for example, 1-2 level LOD models are generated for small-scale object elements such as valves, meters and buttons and 3-5 level $L O D$ models for large-scale object elements such as pumps, motors so as to guarantee the smooth transition, especially, to certain large-scale equipment, firstly it is divided into several sub-model element according to its structural feature (e.g. the main diesel engine can be divided into turbocharger, exhaust manifold, cylinder head and so on) and then establish the $L O D$ models of each sub-model element.

\subsubsection{Structural Nodes Optimization}

This paper optimizes the structural nodes of the geometric model data based on the actual composition and structure, which can not only facility the extracting of the interaction elements, but also quicken the reading and rendering speed of the geometric model data. The detailed optimization process is as follows:

Assume that the original structural nodes of a certain geometric model as Eq.3:

$$
V=\left\{v_{1}, v_{2}, v_{3}, \cdots, v_{i}\right\}
$$

where $V$ is the original structural nodes collection of the geometric model, $v_{l}$ to $v_{i}$ are the $i$ nodes. In this collection, organization of the nodes are in parallel structure and relatively mussy, which is against the extracting of the interaction elements.

The structure of a certain object element in the marine engine room can be expressed in the form of tree hierarchy by analyzing its composition and then the tree hierarchy structure can be expressed in the form of collection as Eq.4:

$$
W=\left\{w_{1}, w_{2}, \cdots, w_{j}\right\}
$$

where $W$ is the structural nodes collection of the tree hierarchy structure, $w_{l}$ to $w_{j}$ are the $\mathrm{j}$ nodes.

The structural nodes optimization process can be seen as the transition process from collection $V$ to collection $W$. Based on the above-mentioned idea, collapse the geometric model in the bottom node of each tree hierarchy's branch during the modeling process and name the collapsed model with real physical meaning, especially, for the interaction elements such as valves and buttons, the dynamic part and static part should be collapsed separately and add interaction identification to the name of the dynamic part so as to be extracted by Unity3D. After completing the collapsing and naming, export the geometric model to Unity3D. The Unity3D is a visual programming tool, which facilities the editing process of the virtual scene greatly. By comparing the collection $V$ and $W$, the original structural nodes of geometric model data will approach the actual tree hierarchy structure gradually with the help of the parenting editing function provided by Unity3D. 


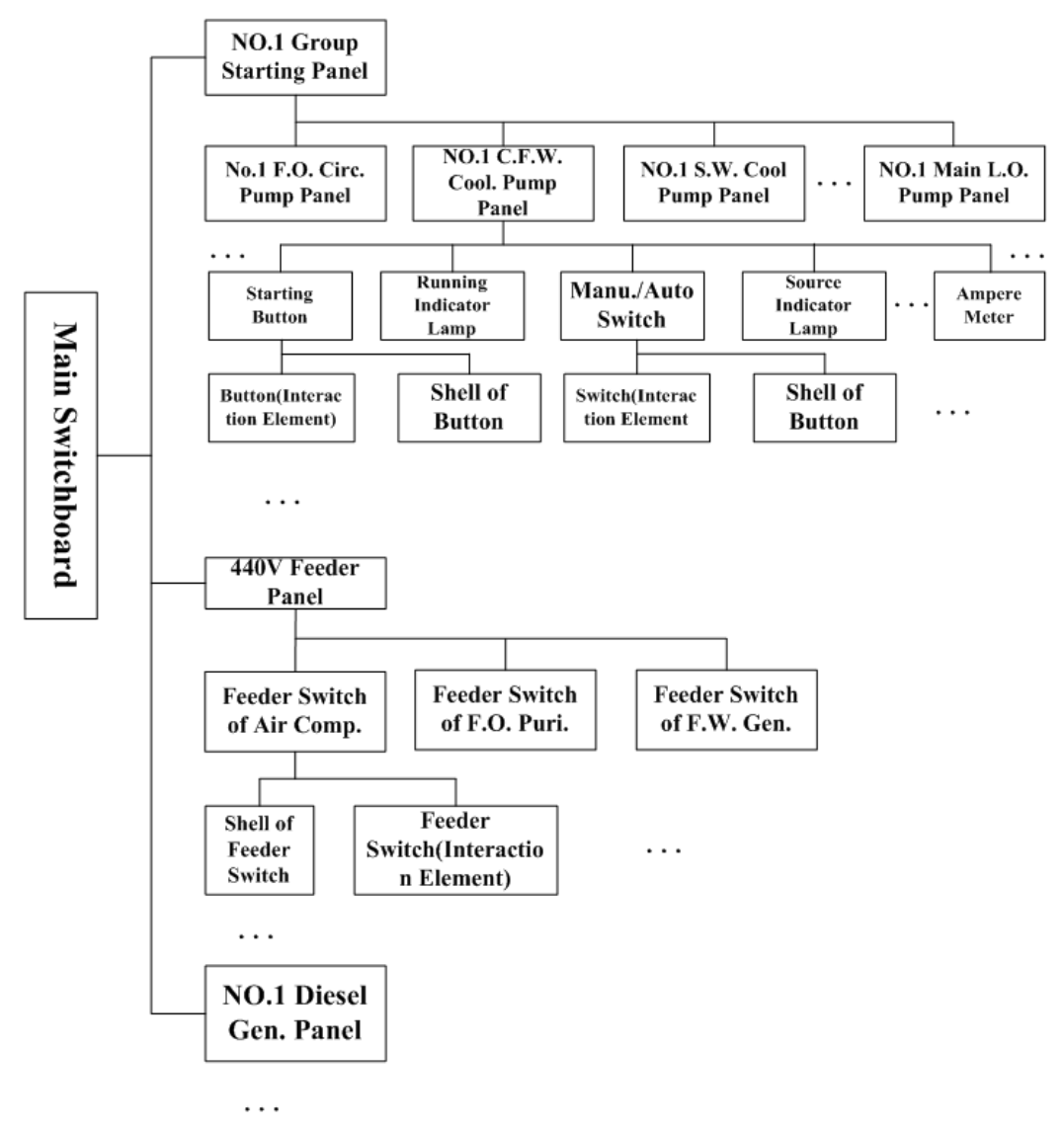

Figure 11. Structural Nodes of Geometric Model Data after Optimization

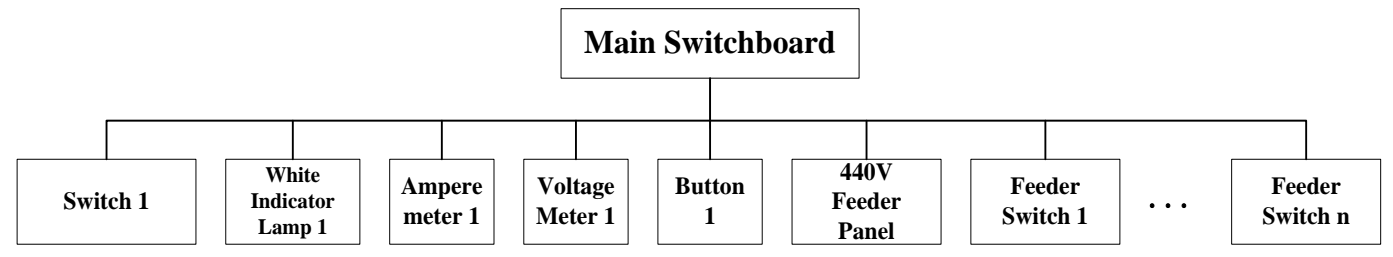

Figure 11. Structural Nodes of Geometric Model Data before Optimization

Figure.11 and Figure.12 take the switchboard as an example to illustrate the optimization result of the structural nodes. It can be found that the organization of the structural nodes of geometric model is more coherent after optimization, which facilities the management of the geometric model data and the extracting of interaction elements.

\subsubsection{Prefab}

During the modeling process, it is found that many model elements have the same appearance and the differences among them are only the position, proportion and orientation. Based on this feature, this paper defines the model element that can be employed universally as Prefab and then arrange them in the right position of the virtual scene after instantiating and adjusting. The Prefab technique can save the memory usage efficiently and improve the simulation efficiency of $3 D$-MERSS. 


\subsection{Rendering Technique}

The virtual scene created by the three-dimensional modeling software only reflects the geometric appearance, which is lack of realistic. So the geometric model of the virtual scene must be rendered so as to reflect the texture and lighting feeling, in this paper two kinds of rendering method, static rendering and Shader, are adopted based on the feature of the marine engine room scene.

\subsubsection{Static Rendering}

Static rendering can be considered that render the geometric model in advance with the static rendering tool (Vray is adopted in this paper) and generate a rendering mapping with integration of material and lighting information, and then apply this rendering mapping to the corresponding geometric model so as to reflect the texture and lighting feeling. The advantage of static rendering is that the texture and lighting information has no need to be calculated during the real-time rendering, which can relieve the calculation load to some extent, and the disadvantage is that a lot of rendering mapping will occupies massive memory space and increase the transmission load between CPU and GPU, meanwhile, the static rendering can only achieve the static lighting effect. So in order to promote the simulation efficiency of $3 D-M E R S S$, static rendering is only applied to certain important equipment.

Especially, for certain clathrate object such as steel grating and ventilation holes, to reflect its appearance feature only by modeling method will not only increase the total number of facets but also lead to undesirable phenomenon such as sawtooth and graphics flicker. Aiming at this problem, this paper uses the mapping with Alpha channel to reflect the clathrate effect. For certain casted objects, the surface of which is usually rough and it is difficult to achieve the desirable effect only by modeling method. Aiming at this problem, this paper uses bump mapping to reflect the rough effect. The bump mapping imposes random disturbance to the normal of the model's surface to make it deviate from the original normal direction, in this way irregular bump effect is achieved. Mapping making software "CrazyBump" is adopted to generate the Alpha mapping and bump mapping in this paper.

\subsubsection{Shader}

With the development of computer graphics card, the concept of programmable pipeline proposed after the release of DirectX 8 permits the developer to control the rendering process through writing the GPU program, and this kind of GPU program is called "Shader" [14]. In virtue of this rendering method, Shader aiming at the marine engine room virtual scene is developed in this paper.

The attenuated Blinn-Phong illumination model is selected to develop the Shader. The Blinn-Phong illumination model considers that the color of a certain vertex on the object surface is composed of ambient light, diffuse reflection and specular highlights reflection, meanwhile, the illumination attenuated effect caused by the distance between the point light and the vertex of object surface is also taken into consideration. The vector representing graph of this attenuated Blinn-Phong illumination model is as shown in Figure.13. 


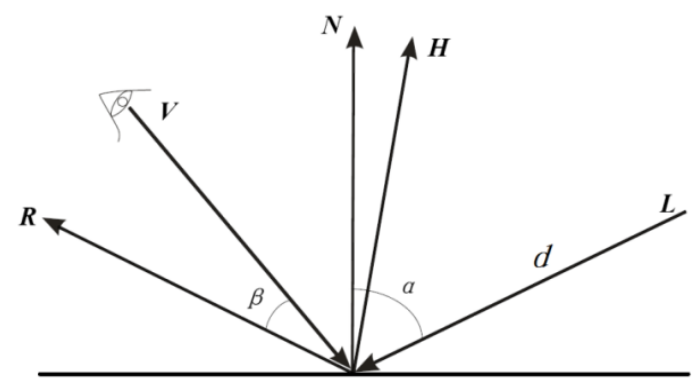

\section{Figure 13. Vector Representing Graph of the Attenuated Blinn-Phong Illumination Model}

In Figure.13, $N$ is the normal vector of the vertex; $L$ is the incident light; $R$ is the reflection light, which is determined by $L$ and $N ; V$ is the observation vector, which is from the observation point to the vertex; $d$ is the distance between the point light and the vertex; $H$ is the bisector of the included angle between $L$ and $V$.

The calculation method of $\mathrm{H}$ is as Eq.5:

$$
H=\frac{(L+V)}{\|L+V\|}
$$

And then the color of the vertex can be got according to Eq.6:

$$
I=A_{i} * A_{c}+k *\left(D_{i} * D_{c} *(N \cdot L)+S_{i} * S_{c} *(N \cdot H)^{n}\right)
$$

Equation.5 is composed of three parts: the first part is the ambient light, which is the color independent of the light source depending on the ambient and the object themself, and the color of ambient light is the same for the same texture, $A_{i}$ is the intensity of ambient light and $A_{c}$ is color of ambient light; the second part is the diffuse reflection, which is the random reflection light of the incident light in all directions determined by the included angle between $L$ and $N, D_{i}$ is the intensity of diffuse reflection and $D_{c}$ is the color of diffuse reflection; the third part is the specular highlights reflection, which is the definite reflection light of the incident light in certain direction determined by the smooth degree of the object surface and $H, S_{i}$ is the intensity of specular highlights reflection, $S_{c}$ is the color of specular highlights reflection, $\mathrm{n}$ is glossiness of the object surface, and the bigger the $\mathrm{n}$ is, the more smooth the object's surface is; $k$ is the attenuated coefficient and it is the reciprocal number of the quadratic polynomial about the propagation distance $d$, the calculation method of $\mathrm{k}$ is as Eq.7:

$$
f(d)=\frac{1}{a_{2} * d^{2}+a_{1} * d+a_{0}}
$$

Shader editing tool provided by Unity3D is used to write the Shader, which is applied to a lot of model elements such as pipes, bulkhead and floors by adjusting the parameters of the illumination model. As the texture and illumination effect is completely generated by GPU, the calculation load of CPU will be eased and the simulation efficiency of 3DMERSS will be promoted in virtue of the strong parallel processing capability of GPU.

The marine water chilling unit is used to test the rendering effect as the Figure.14 shows and it is found that better visual effect can be achieved by using cooperatively the above-mentioned rendering method. 


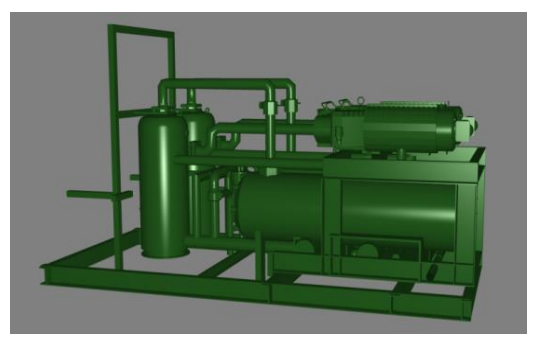

(a) Attenuated Blinn-Phong Lighting Model
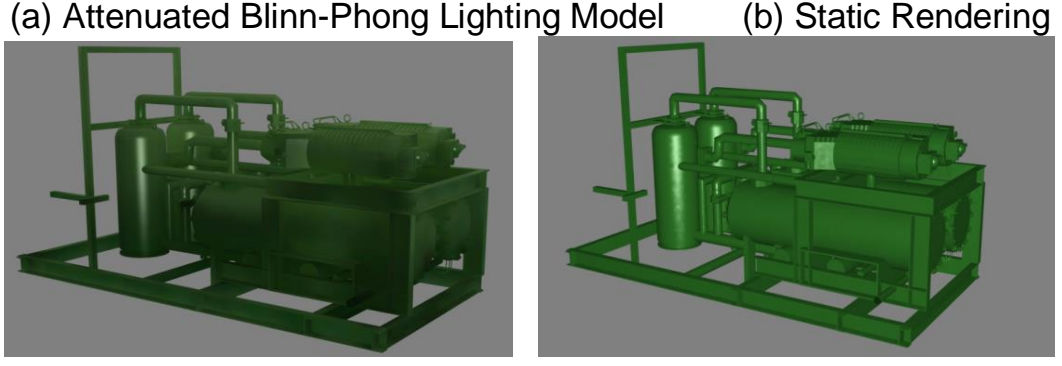

(c) Attenuated Blinn-Phong Lighting Model + Static Rendering (d) Attenuated Blinn-Phong Lighting Model + Bump Texture

\section{Figure 14. Comparison Diagram of the Rendering Effects}

\subsubsection{Channel Stereo Projection Environment for the 3D Marine Engine Room Simulation Software}

To improve the user's experience, this paper also applied the $3 D$-MERSS to the 3channel stereo projection environment besides the desktop environment. The 3-channel stereo projection environment belongs to immersive $V R$ system and the users can feel the depth perception strongly [15].

The composition and structure of the 3-channel stereo projection environment is as the Figure.15 shows:

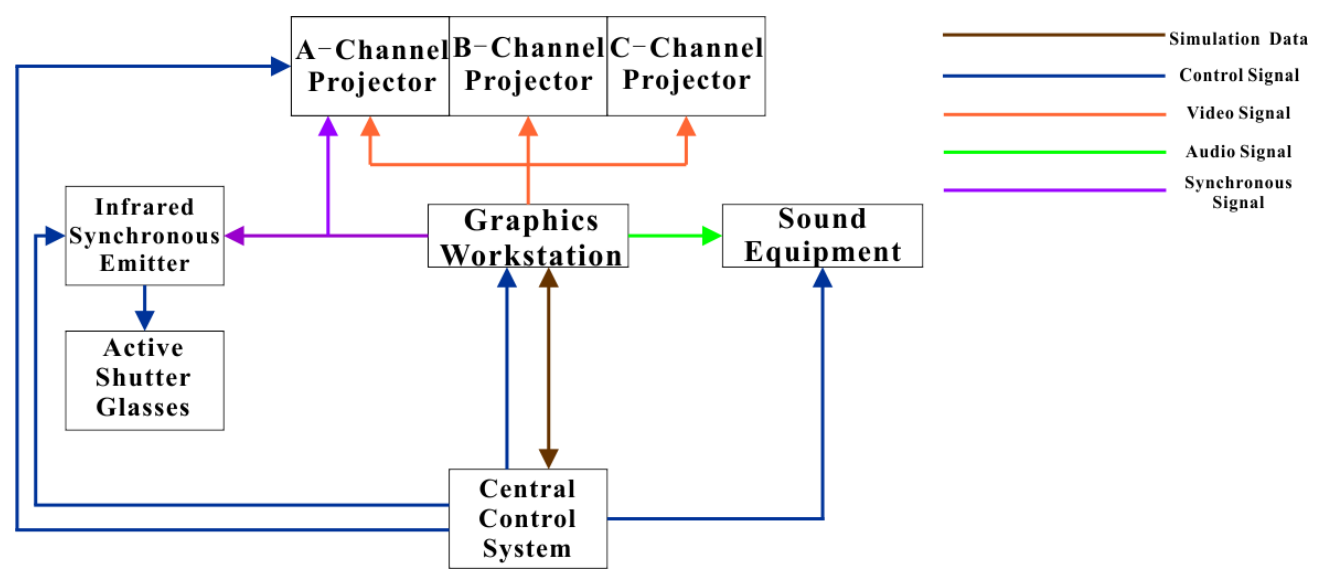

\section{Figure 15. Composition and Structure of the Three-Channel Stereo Projection Environment}

In Figure.15, the central control system is in charge of the other parts and the running of $2 D-M E S S S$; the graphic workstation is the in charge of the real-time rendering of the marine engine room virtual scene and outputting video signal to projector A, B and C. The infrared synchronous emitter sends the switching signal to the active shutter glasses to simulate the visual principle of the human eye. In this way, depth information of virtual scene is generated in the brain of the user and strong immersion is achieved. 
During establishing the 3-channel stereo projection environment for the 3D-MERSS, the main related key techniques include scene segmentation, geometric correction and edge blending.

\subsubsection{Scene Segmentation}

As the virtual scene is completely generated by one single graphic workstation, so the video signal for each projector is got by segmenting the frame buffer graphics in this paper. Compared with the multi-screen conjoint technique, blending region with a certain width should be reserved when segmenting the frame buffer graphics as the Figure.16 shows, in this way it can guarantee the consistency and integrity of the projection graphics after blending.
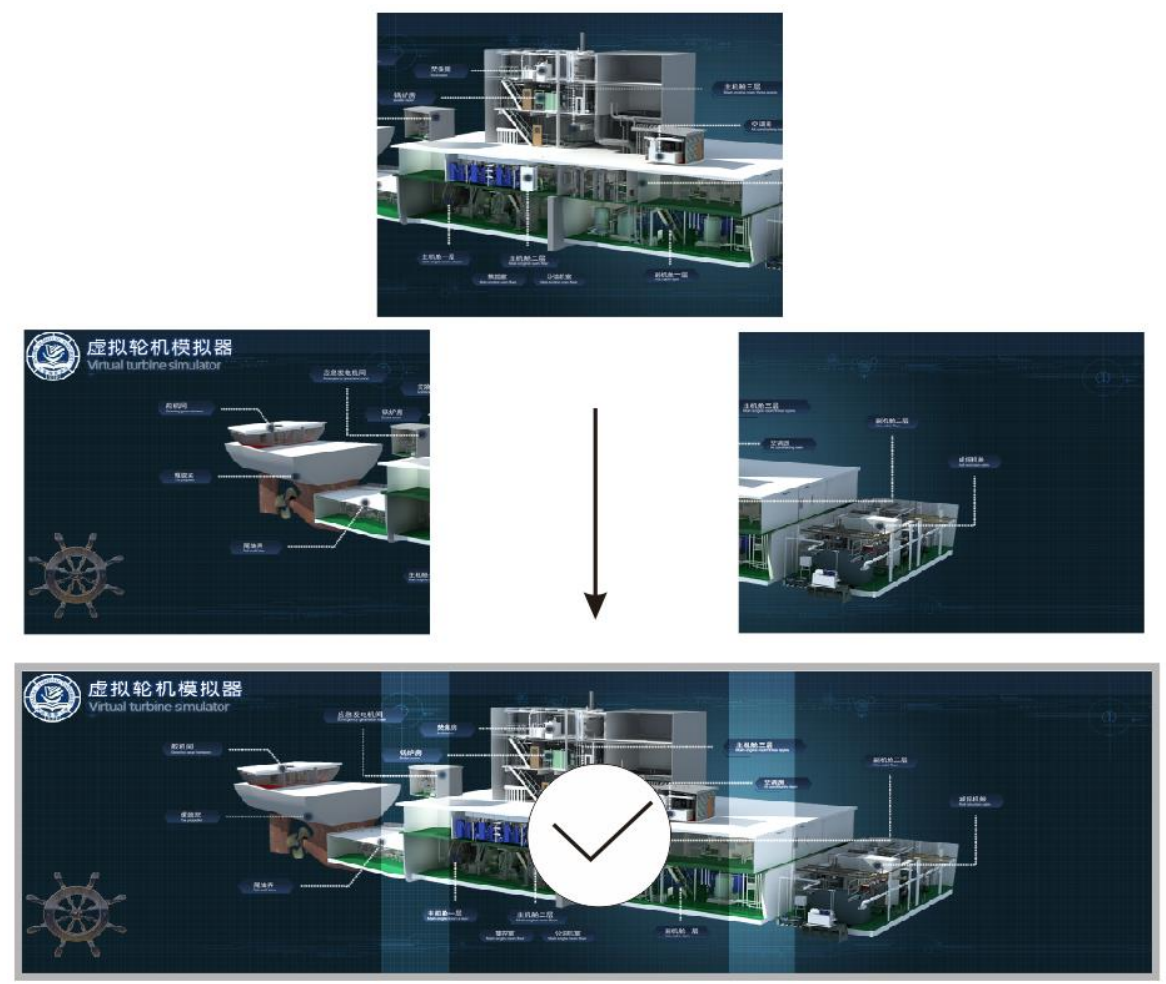

Figure 16. Scene Segmentation with Blending Region

\subsubsection{Geometric Correction}

The geometric correction mainly involves raster alignment and deformation correction. Raster alignment means adjusting the projection raster to make the conjoint part of the adjacent channel's graphic consistent. The deformation correction involves keystone correction and nonlinear distortion correction.

As it is usually difficult to guarantee that the projector keeps vertical with the projection screen strictly only by adjusting the position of the projector, so the keystone distortion will happen. The keystone distortion involves horizontal keystone distortion and vertical keystone distortion and there're two correction methods for the keystone distortion, which are optical keystone correction and digital keystone correction. Optical keystone correction means adjusting the physical position of the projector lens to achieve the goal of keystone correction and digital keystone correction means eliminating the keystone distortion completely by the correction software. At present, with the development of projector technology, almost all of the projectors have been integrated 
with the function of keystone correction, which will adjust the shape of the graphics before displaying by interpolation algorithm.

At present, the nonlinear distortion correction of the planar projection environment has been solved very well and the current research direction mainly targets the nonlinear distortion correction algorithm of the curved projection environment. The typical algorithm includes the matrix mapping method and polynomial function correction method proposed by Raskar [16], self-calibration method proposed by Hartley and Faugers [17], the flexible camera calibration method proposed by Zhang [18].

This paper adopts the nonlinear distortion correction algorithm based on Bezier curve proposed by Zeng [19]. The basic idea of this algorithm: The coordinate calculation process of the deformed surface's grid points can be seen as the construction process of surface and a curved line can be generated quickly based on the three-order Bezier curve through a group of multilateral line as the Eq.8 shows, and thus the boundary of the deformed screen can be generated through four Bezier curves. The coordinates of the boundary are used to generate grids, which are used in texture mapping and the raster alignment of blending region. The calculation method of grid coordinates is shown in Eq. 9 and Eq.10. The Eq.9 shows that the horizontal coordinate of the deformed grid node is composed of the original gird node's horizontal coordinate and two correction terms. The first correction item is superimposing lateral deviation of upper boundary grid nodes relative to the original position on each node of the same column in the way of linear decreasing and the second correction item is superimposing lateral deviation of lower boundary grid nodes relative to the original position on each node of the same column in the way of linear increasing. The calculation of the vertical coordinate of the deformed grid node is as similar as the horizontal coordinate.

$$
P(u)=P_{1}(1-u)^{3}+3 C_{1}(1-u)^{2} u+3 C_{2}(1-u) u^{2}+P_{2} u^{3}
$$

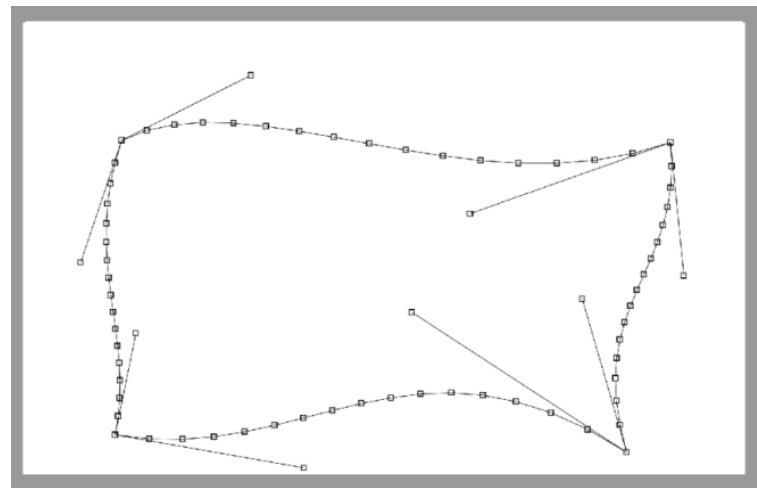

Figure 17. Boundary of Deformed Screen Generated by Bezier Curve

$$
\begin{gathered}
P_{i j}^{\prime} \cdot x=P_{i j} \cdot x+\left(P_{0 j}^{\prime} \cdot x-P_{0 j} \cdot x\right) \cdot\left(1-\frac{i}{M}\right)+\left(P_{M j}^{\prime} \cdot x-P_{M j} \cdot x\right) \cdot \frac{i}{M} \\
P_{i j}^{\prime} \cdot y=P_{i j} \cdot y+\left(P_{i 0}^{\prime} \cdot y-P_{i 0} \cdot y\right) \cdot\left(1-\frac{j}{N}\right)+\left(P_{i N}^{\prime} \cdot y-P_{i N} \cdot y\right) \cdot \frac{j}{N}
\end{gathered}
$$

The Figure.18 shows the grid comparison before and after correction and various shapes of free-form surface can be got only by adjusting 12 control points. 


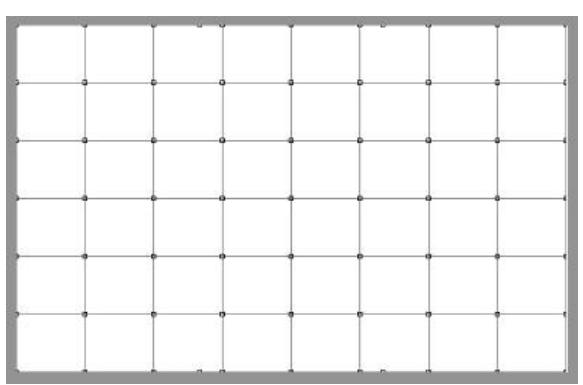

(a) Grid before Correction

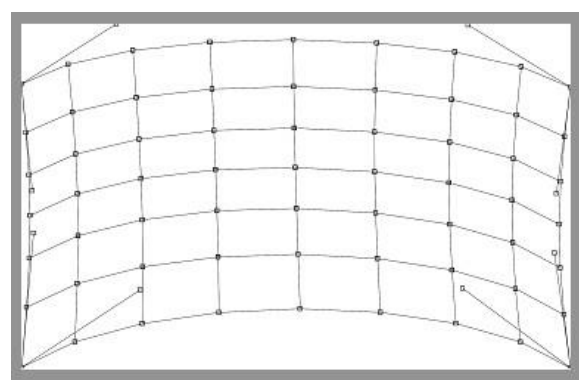

(b) Grid after Correction

Figure 18. Comparison before and after Correction

\subsubsection{Edge Blending}

Although the blending region is taken into consideration during scene segmentation in this paper, the luminance of the blending region is obviously different from the other parts after graphics stitching as the Figure.16 shows, so the luminance of the frame buffer graphics' blending region should be adjusted by applying blending algorithm. The essence of blending algorithm is respectively adjusting the pixel value of overlapping region between the left and right channel as so to make the luminance of projection graphics consistent. To eliminate the luminance difference of the blending region as far as possible and enhance the flexibility of blending algorithm in the practical application, blending function based on cubic spline curve is adopted in this paper.

The spline curve is a kind of curve that is generated by a group of control points and the shape of the curve is completely controlled by these control points, that is to say, arbitrary shapes of curve can be got by adjusting these control points.

The cubic spline curve $S(x)$ is a segmented function and $n+1$ data points $\left(x_{0}, y_{0}\right),\left(x_{1}\right.$, $\left.y_{1}\right) \ldots\left(x_{n}, y_{n}\right)$ are given. The cubic spline curve $S(x)$ should meet the following conditions:

(1) $S(x)=S_{i}(x)$ is cubic polynomial in each segmentation interval $\left[x_{i}, x_{i+1}\right](i=0,1, \ldots, n-1)$.

(2) $S(x)$ should satisfy the condition $S\left(x_{i}\right)=y_{i}(i=0,1, \ldots, n)$.

(3) The first derivative and second derivative function of $S(x)$ are both continuous in the interval $\left[x_{0}, x_{n}\right]$, that is to say, the cubic spline curve $S(x)$ is smooth.

Therefore, the segmented function $S_{i}(x)$ can be expressed as Eq.11:

$$
S_{i}(x)=a_{i}+b_{i}\left(x-x_{i}\right)+c_{i}\left(x-x_{i}\right)^{2}+d_{i}\left(x-x_{i}\right)^{3}, i=0,1, \cdots n-1
$$

where $a_{i}, b_{i}, c_{i}, d_{i}(\mathrm{i}=0,1, \ldots, \mathrm{n}-1)$ are $4 n$ undetermined coefficients.

According to the above-mentioned analyzed, the following results can be got:

(1) Function Continuity and Interpolation Character

$$
S_{i}\left(x_{i}\right)=y_{i}, S_{i}\left(x_{i+1}\right)=y_{i+1}, i=0,1, \cdots, n-1
$$

Due to $S_{i}\left(x_{i}\right)=y_{i}$, Eq.13 can be got:

$$
a_{i}=y_{i}, i=0,1, \cdots, n-1
$$

Define step size $h_{i}=x_{i+1}-x_{i},(\mathrm{i}=0,1, \ldots, \mathrm{n}-1)$ and substitute $h_{i}$ into $S_{i}\left(x_{i+1}\right)=y_{i+1}(i=0,1, \ldots, n$ 1), and then Eq.14 can be got:

$$
a_{i}+h_{i} b_{i}+h_{i}^{2} c_{i}+h_{i}^{3} d_{i}=y_{i+1}, i=0,1, \cdots, n-1
$$

(2) Differential Continuity

$$
S_{i}{ }^{\prime}\left(x_{i+1}\right)=S_{i+1}{ }^{\prime}\left(x_{i+1}\right), S_{i}^{\prime \prime}\left(x_{i+1}\right)=S_{i+1}{ }^{\prime \prime}\left(x_{i+1}\right), i=0,1, \cdots, n-2
$$

Due to the function expression of each segmentation of $S(x)$, Eq.16 and Eq.17 can be got:

$$
S_{i}^{\prime}(x)=b_{i}+2 c_{i}\left(x-x_{i}\right)+3 d_{i}\left(x-x_{i}\right)^{2}
$$




$$
S_{i}^{\prime \prime}(x)=2 c_{i}+6 d_{i}\left(x-x_{i}\right)
$$

Due to $S_{i}^{\prime}\left(x_{i+1}\right)=S_{i+1}{ }^{\prime}\left(x_{i+1}\right), i=0,1, \cdots, n-2$ and the step size hi, Eq.18 can be got:

$$
b_{i}+2 h_{i} c_{i}+3 h_{i}^{2} d_{i}-b_{i+1}=0, i=0,1, \cdots, n-2
$$

Due to $S_{i}^{\prime \prime}\left(x_{i+1}\right)=S_{i+1}{ }^{\prime \prime}\left(x_{i+1}\right), i=0,1, \cdots, n-2$ and the step size hi, Eq.19 can be got:

$$
2 c_{i}+6 h_{i} d_{i}-2 c_{i+1}=0, i=0,1, \cdots, n-2
$$

Assume that $m_{i}=S_{i}^{\prime \prime}\left(x_{i}\right)=2 c_{i}$ and then $c_{i}=\frac{m_{i}}{2}$ can be got, substitute ci into Eq.19 and then $d_{i}=\frac{m_{i+1}-m_{i}}{6 h_{i}}$ $b_{i}=\frac{y_{i+1}-y_{i}}{h_{i}}-\frac{h_{i}}{2} m_{i}-\frac{h_{i}}{6}\left(m_{i+1}-m_{i}\right)$ can be got, substitute ci and di into Eq.18 and then Eq.20 can be got:

$$
h_{i} m_{i}+2\left(h_{i}+h_{i+1}\right) m_{i+1}+h_{i+1} m_{i+2}=6\left[\frac{y_{i+2}-y_{i+1}}{h_{i+1}}-\frac{y_{i+1}-y_{i}}{h_{i}}\right]
$$

According to the value range of $i$, it is known that $n-1$ equations exists, however, $n$ unknown quantities exist and thus two extra equations about mi are needed to solve the equation set, so the interval endpoints $x_{0}$ and $x_{n}$ are usually needed to be restricted. Restriction method based on free boundary are adopted in this paper, that is to say, the two ends are not under any force that makes it curve, which means $m_{0}=S_{0}{ }^{\prime \prime}\left(x_{0}\right)=0$ and $m_{n}=S_{n}^{\prime \prime}\left(x_{n}\right)=0$, and thus Equation.19 can be expressed in the form of matrix equation as the Eq.21 shows:

$$
\left[\begin{array}{cccccc}
1 & 0 & 0 & & \ldots & 0 \\
h_{0} & 2\left(h_{0}+h_{1}\right) & h_{1} & 0 & \cdots & \\
0 & h_{1} & 2\left(h_{1}+h_{2}\right) & h_{2} & \ldots & \\
0 & 0 & h_{2} & 2\left(h_{2}+h_{3}\right) & h_{3} & \vdots \\
\vdots & & \ddots & \ddots & \ddots & \\
0 & \cdots & & h_{n-2} & 2\left(h_{n-2}+h_{n-1}\right) & h_{n-1} \\
m_{2} \\
m_{3} \\
\vdots \\
m_{n}
\end{array}\right]=6\left[\begin{array}{c}
0 \\
\frac{y_{2}-y_{1}}{h_{1}}-\frac{y_{1}-y_{0}}{h_{0}} \\
\frac{y_{3}-y_{2}}{h_{2}}-\frac{y_{2}-y_{1}}{h_{1}} \\
\frac{y_{4}-y_{3}}{h_{3}}-\frac{y_{3}-y_{2}}{h_{2}} \\
\vdots \\
\frac{y_{n}-y_{n-1}}{h_{n-1}}-\frac{y_{n-1}-y_{n-2}}{h_{n-2}} \\
0
\end{array}\right]
$$

According to Eq.21, $n+1$ unknown quantities $m_{i}(i=0,1, \ldots, n)$ can be solved and thus the cubic spline curve is got. Figure. 19 shows the spline based on 7 data points and different shapes of curve can be got by adjusting the position of data points, and thus the luminance transition process of different regions in the blending region can be adjusted. Figure.20 is the actual blending effect of the Anchor and Mooring Equipment Operation Room and it can be found that the luminance of the whole projection screen is consistent, and thus the effectiveness of the blending algorithm proposed in this paper is verified. 


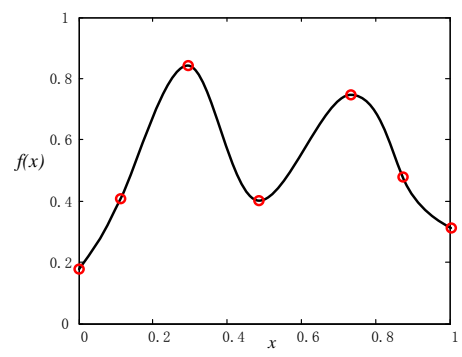

Figure 19. Cubic Spline Curve Based on 7 Data Points

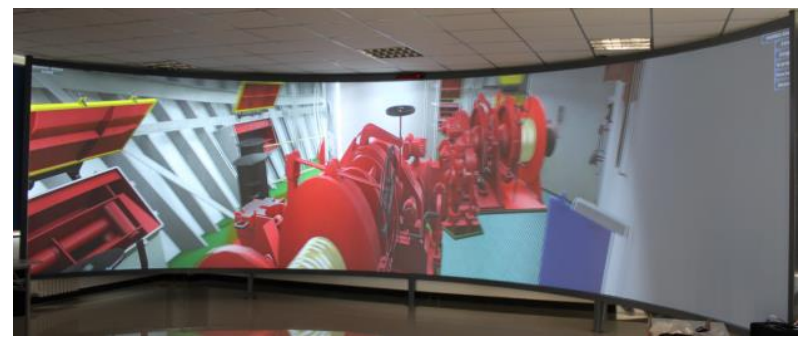

Figure 20. Blending Effect of Anchor and Mooring Equipment Operation Room

\section{Conclusion}

AHTS-vessel "HYSY 681" is selected as the parent ship to develop the AHTS-Vessel MERVTP. The development procedure and the related key techniques are introduced in detail in this paper and conclusions are got as follows:

(1) Design mode of mixed simulation methods including $2 D, 3 D$ and semi-physical simulation ways is adopted to establish the AHTS-Vessel MERVTP, which can make full advantages of each simulation method. The 2D-MERSS can display the principle of marine engineering system and the changing trend of state parameter clearly and intuitively and it also has the feature of easy operation; The 3D-MERSS can display the structure and appearance of the marine engine room and corresponding equipment intuitively, which also supports virtual roaming and human-machine interaction; The physical simulation consoles are designed and manufactured completely in accordance with the real one, which can provide the users real manipulation feeling.

(2) The simulation data is planned from an overall point of view and in this way it can facility the management, invoking, transmission and identification of the simulation data; Select the proper simulation algorithm and strategy so as to balance the requirement on instantaneity and precision.

(3) The utilization of $L O D$ and Prefab technique can improve the fluency of scene graphics effectively; Optimize the structural nodes of the geometric model data can not only facility the extracting of interaction elements by Unity3D, but also quicken the reading and rendering speed of geometric model; Applying static rendering and Shader jointly can ease the calculation load of $\mathrm{CPU}$ and improve the real-time rendering efficiency on the basis of guarantee the realistic of the virtual scene.

(4) Applying the 3D-MERSS to the 3-channel stereo projection environment can promote the immersion of the virtual scene, in addition, the scene segmentation, edge blending and geometric correction method proposed in this paper have fine practical effect.

The AHTS-Vessel MERVTP has already been applied in the Ship Division in COSL (China Oilfield Services Limited) and achieved good training effect. In the subsequent 
research work, the research emphasis will focus on the online edition marine engine room training platform.

\section{References}

[1] H.S. Shen, J.D. Zhang, H. Cao, "Application and Development of Marine Engine Room 3-D Visual Simulation System”, China Water Transport, vol. 16 (2016), pp.66-70.

[2] R. Laskowski, L. Chybowski, K. Gawdziska, "An Engine Room Simulator As a Tool for Environmental Education of Marine Engineers", World Conference on Information Systems and Technologies, Azores, Portugal, (2015) April 1-3.

[3] http://www.unitest.pl/

[4] http://www.km.kongsberg.com/

[5] Y.L. He, H. Chen, "Research on Visual Simulation System of Electric Propulsion Simulator", Journal of Wuhan University of Technology (Transportation Science \& Engineering), vol. 36 (2012), pp.12041208.

[6] http://www.cnooc.com.cn/data/html/news/2011-06-01/chinese/302188.html

[7] J.F. Chen, H.N. Hsieh, Q.H. Do "Evaluating Teaching Performance Based on Fuzzy AHP and Comprehensive Evaluation Method", Applied Soft Computing Journal, vol. 28 (2015), pp.100-108.

[8] Y. Lin, J. Chen, Y. Liu, Y.T. Wang "User Experience Design of VR-AR Hybrid Mobile Browsing System Based on Mental Mode", Chinese Journal of Computers, vol. 28 (2015), pp.408-422.

[9] H.B. Gan, G. Ren, J.D. Zhang, "Design and Implementation for LNG Carrier Propulsion Simulation System", Shipbuilding of China, vol. 53(2012), pp.146-156.

[10] S. Timothy, "Numerical Analysis", Addison Wesley Longman, England, (2011).

[11] A. Kozminski, "Windows Presentation Foundation (WPF) Technology Meets the Challenges of Operator Interface Design in Automatic Test Systems", 48th IEEE Systems Readiness Technology Conference, Anaheim, CA, United States, (2012) September 10-13.

[12] J. Zhao, "Adaptive Optimization of LOD Algorithm on Real-time Rendering of 3D Urban Planning", International Journal of Earth Science and Engineering, vol. 7 (2014), pp.2554-2559.

[13] W. Shangguan, H.Q. Guo, P.H. Liu, B.G. Cai, J. Wang, "Visual Simulation Model of CVIS", Journal of Traffic and Transportation Engineering, vol. 15(2015), pp.118-126.

[14] Z. Zuo, Y. He, Y.L. Duan, Q.B. Li, J.M. Feng, L.Y. Zhang, "Scientific Visualization of Arch Dam Projects Based on HTML5 Standards", Journal of Computer-Aided Design \& Computer Graphics, vol. 26(2014), pp.590-596.

[15] H. Zeng, J.D. Zhang, G. Ren, H.B. Gan "Design and Implementation of Marine Engine Room Three Dimensional Visual Simulation System", Journal of System Simulation, vol.26(2014), pp.363-368+375.

[16] R. Raskar, B.J. Van, "Low-cost Multi-Projector Curved Screen Displays", SID Symposium Digest of Technical Papers, vol. 36(2005), pp.884-887.

[17] I.H. Richard, "Estimation of Relative Camera Positions for Uncalibrated Cameras", Lecture Notes in Computer Science, vol. 588(2005), pp.579-587

[18] Z.Y. Zhang, “A Flexible New Technique for Camera Calibration”, IEEE Transactions on Pattern Analysis and Machine Intelligence, vol. 22(2000), pp.1330-1334.

[19] H. Zeng, J.D. Zhang, L.S. Ma, B.Z. Jia, "Fast Multi-projection Image Geometric Calibration and Edge Blending Method", Computer Engineering and Design, vol. 34(2013), pp.1846-1850.

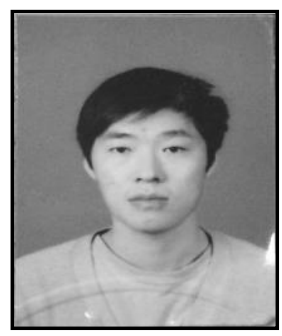

Haosheng Shen, received his B.S. and M.S. degrees in Marine Engineering from Dalian Maritime University, Dalian, China, in 2011 and 2013, respectively. He is currently Ph.D. candidate majored in Marine Engineering in Dalian Maritime University. His research interests include visualization simulation including $2 \mathrm{D}$ and $3 \mathrm{D}$, math modeling and simulation of marine diesel engine. 


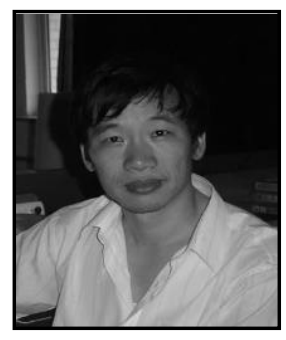

Jundong Zhang, received his B.S. and M.S. degrees in Marine Engineering from Dalian Maritime University, Dalian, China, in 1989 and 1992, respectively, and Ph.D. degree in Marine Engineering from Dalian Maritime University, in 1998. His research interests include computer and network application, marine engineering automation and control, modeling and simulation of marine engineering system.

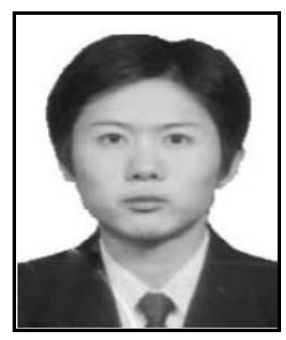

Hong Zeng, received his B.S. and M.S. degrees in Marine Engineering from Dalian Maritime University, Dalian, China, in 1998 and 2007, respectively, and Ph.D. degree in Marine Engineering from Dalian Maritime University, in 2012. His research interests include software engineering and application, 3D Visual Simulation Technology.

Yuanyuan Tang, received his B.S. and M.S. degrees in Marine Engineering from Dalian Maritime University, Dalian, China, in 2012 and 2014, respectively. He is currently Ph.D. candidate majored in Marine Engineering in Dalian Maritime University. His Research interests include WPF technology, math modeling and simulation of marine diesel engine. 
International Journal of Multimedia and Ubiquitous Engineering Vol.12, No.4 (2017) 